\title{
Guest-editorial
}

\section{Special issue on agent-mediated knowledge management}

\author{
Jurriaan van Diggelen and Virginia Dignum \\ Department of Information and Computing Sciences, Utrecht University, The Netherlands \\ E-mail: \{jurriaan,virginia\}@cs.uu.nl
}

\section{Introduction}

The main goal of Knowledge Management (KM) is to provide relevant knowledge to assist users in executing knowledge intensive tasks. That is, KM aims at facilitating an environment where work critical information can be created, structured, shared, distributed and used. To be effective, KM must provide users with relevant knowledge, at the right time and in the right form, that enables users to better perform their tasks. Knowledge Management (KM) has been a predominant trend in business in the recent years.

Though KM is primarily a management discipline (with a background in human resource management, strategy, and organizational behavior), the role of information technology as an enabling factor is widely recognized, and - after a first phase where merely general purpose technology like Internet/Intranets or email were found to be useful to facilitate KM - variety of proposals exist to support KM with specialized information systems [3]. Often, IT research for KM focused on the comprehensive use of an organization's knowledge, thus aiming at the completeness of distribution of relevant information. Technically, this is typically supported by centralized approaches: knowledge about people, processes or domain knowledge is represented and maintained in global repositories which serve as sources to meet a knowledge worker's (potentially complex) information needs. Such repositories may be structured by global ontologies (e.g., in form of knowledge portals) or they may be rather flat and processed by weak (i.e. not knowledge-intensive) methods like statistics-based information retrieval or collaborative filtering.

However, as is often mentioned in the literature, knowledge tasks have a collaborative aspect, that is, an individual can best acquire and use knowledge by making use of existing relations among people (communities) or by reusing and personalizing information already collected and annotated by others. Furthermore, a KM system must be able to adapt to changes in the environment, to the different needs and preferences of users, and to integrate naturally with existing work methods, tools and processes. That is, KM systems must be reactive (able to respond to user requests or environment changes) and proactive (able to take initiatives to attend to user needs). These aspects also characterise intelligent software agents, what seems to indicate the applicability of agent technology in the KM area.

Intelligent agents as a paradigm for developing software applications are currently the focus of intense interest on the part of many fields of computer science and artificial intelligence. A software agent is an autonomous entity that perceives and acts on its environment in order to achieve its goals. Wooldridge and Jennings [4] defined four properties that form a weak definition of Agency:

- autonomy: acting without direct intervention of people or other systems.

- social ability: ability to interact with other agents (and possibly with people). 
- reactivity: perception of environment, and ability to respond to changes in that environment.

- pro-activeness: can act from its own initiative (in order to reach own goals) rather then wait for reactive processing.

Autonomous agents are capable of interacting with other agents using a specific communication language, thus creating a sort of social ability that allows them to perceive their environment, respond to its changes or achieve goals by simply adapting and reacting to other players. A Multi-Agent System (MAS) can therefore be defined as: "a collection of possibly heterogeneous, computational entities, having their own problem solving capabilities and which are able to interact among them in order to reach an overall goal" [2].

Agents usually operate in a dynamic, nondeterministic and complex environment in which a single input action often produces unexpected results. MAS environments assume no global control, data decentralization and asynchronous computation. Furthermore, agents in a MAS are assumed to operate with incomplete information or capabilities for solving the problem. Communication is thus the key for agents to share the information they collect, to co-ordinate their actions and to increase inter-operation. In heterogeneous systems, knowledge sharing is hampered by the lack of common ontologies. Therefore, adequate support for ontology matching and meaning negotiation is of great importance to MAS and to AMKM in special.

\section{Agents in knowledge management}

Current developments in $\mathrm{KM}$ indicate a need for systems that are reactive and proactive in relation to the needs and expectations of their users. Based on the characteristics of KM systems and the weak definition of agents, recent research developments claim that agent-based approaches are especially well - suited to support KM with information technology. A KM environment can be described as a distributed system where different agents, acting autonomously on behalf of a user and each pursuing its own goals, need to interact in order to achieve their goals. In such environments, the ability to communicate and negotiate is paramount. Furthermore, the number and behaviour of participants cannot be fixed a priori and the system can be expected to expand and change during operation, both in number of participants and in amount or kind of knowledge shared. The choice for multi-agent systems for KM is motivated by the following observations:
- KM domains involve an inherent distribution of data, problem solving capabilities and responsibilities (conforms to the ideas of autonomy and social ability of agents).

- The integrity of the existing organisational structure and the autonomy of its subparts need to be maintained (uses autonomous nature of the agents).

- Interactions in KM environments are fairly sophisticated, including negotiation, information sharing, and coordination (requires complex social skills with which agents are endowed).

- KM domains call for a functional separation between knowledge use and knowledge sources as a way to incorporate dynamic behaviour into information systems design (agents can act as mediators between source and application of knowledge).

- The solution for KM problems cannot be entirely prescribed from start to finish and therefore problem solvers are required to respond to changes in the environment, to react to the unpredictability of business processes and to proactively take opportunities when they arise (requires the reactive and proactive abilities of agents).

Agent-Mediated Knowledge Management (AMKM) proposes agent-based approaches to deal with collective aspects of the domain in an attempt to cope with the conflict between desired order and actual behavior in dynamic environments. Van Elst et al. argued that "the basic features of agents (social ability, autonomy, reand pro-activeness) can alleviate several of the drawbacks of the centralized technological approaches for $K M$ " [3]. Reactivity and proactivity of agents help to cope with the flexibility needed to deal with the complex nature of KM tasks. The resulting complex interactions with the related actors in the KM landscape and the environment can be supported and modeled by the complex social skills with which agents can be endowed. Autonomy of agents enables to represent the reality that knowledge workers typically do not adopt KM goals with a high priority, and that KM activities are incorporated into their daily tasks differently by different people.

In complex environments, the flow of knowledge within an organisation (or organisations) must take into account not only the knowledge needs of business processes, but also the personal preferences and level of expertise of individual users. Agents can, on the one hand, lead to advanced functionality of KM systems, e.g. personalisation of knowledge presentation and matching supply and demand of knowledge. On the other hand, 
the rich representational capabilities of agents as modelling entities allow for more faithful and effective treatments of complex organisational processes.

\section{The role of ontologies in agent based KM systems}

Because communication is one of the core aspects in KM systems, it is crucial for the agents to have mutual understanding about how meanings are expressed in messages. This is enabled by an ontology which is usually thought of as a way to specify content specific agreements [1]. Agents that have adopted the same ontology have no difficulty in sharing knowledge as the common ontology guarantees that the receiver's interpretation of a message corresponds to what the sender intended to convey with it.

As agent based KM systems are often heterogeneous, a common ontology is usually absent, which poses problems for knowledge sharing. The most obvious way to deal with these communication problems would be to develop one common ontology and enforce it upon every agent. However, this solution is very difficult to apply in practice, as it requires all involved system developers to reach consensus on which ontology to use. Moreover, it requires each agent to abandon its own "world view" and adopt an ontology that is not specifically tailored to its own task. Such a solution would be in conflict with the agent's autonomy.

For these reasons, more sophisticated techniques have been proposed that allow agents to preserve their own ontology and enable the agents to communicate using ontology mappings. Ideally, these ontology mappings are established automatically. As this is often not feasible, many approaches require human intervention to find these mappings. A debatable issue is whether the ontology mappings should be established only once, at design time, or that it should also be possible to establish new mappings at run-time. Also, the system must be structured in such a way, that the agents know how to deal with ontology problems. Should every agent be capable of solving ontology mismatches, or should this task be left to specialized agents that serve as ontology mediators?

The challenge for KM systems is to provide a feasible solution for ontology problems that does not unnecessarily restrict the agent's autonomy and freedom to use its own ontology.

\section{Overview}

This issue contains three extended papers from the third international workshop on Agent Mediated Knowledge Management (AMKM 2005) and one from MICAI 2005. The papers were separately reviewed, and judged on their quality, significance and originality. This resulted in the four high quality papers that are included in this special issue.

The first paper, entitled "Solving Power and Trust Conflicts using Argumentation in Agent-mediated Knowledge Distribution" by C.I. Chesñevar, R. Brena and J.L. Aguirre, describes an approach for distributing knowledge and information using defeasible logic programming. The framework incorporates power relations, delegation and trust.

The second paper, called "A Framework for Distributed Knowledge Management in Autonomous Logistic Processes" by H. Langer, J.D. Gehrke, J. Hammer, M. Lorenz, I.J. Timm, O. Herzog, presents an approach to enable robust decision-making in a multiagent environment. The framework is applied to the logistics domain.

The third paper, called "Building Common Awareness in Agent Organizations", by I. Partsakoulakis and G. Vouros, describes an approach for reconciling different views of the world and deciding on a single view that every agent within the group accepts. Their framework is applied to asynchronous backtracking.

The last paper, entitled "Analysis and Evaluation of Distributed Knowledge Management by AgentBased Simulation" by $M$. Wurst, presents an evaluation method for intelligent mediation techniques using agent-based simulation. The framework is applied to collaborative filtering and the ontology matching problem.

\section{References}

[1] T. Gruber, A Translation Approach to Portable Ontology Specifications, Knowledge Acquisition 5 (1993), 199-220.

[2] J. Ferber, Multi-Agent System: An Introduction to Distributed Artificial Intelligence, Addison Wesley Longman, 1999.

[3] L. van Elst, V. Dignum and A. Abecker, Towards AgentMediated Knowledge Management, in: Agent-Mediated Knowledge Management: Selected Papers, L. van Elst, V. Dignum and A. Abecker, eds, LNAI 2926, Springer, 2004.

[4] M. Wooldridge and N.R. Jennings, Intelligent Agents: Theory and Practice, Knowledge Engineering Review 10(2) (1995). 